\title{
PCSK9 inhibitors - Clinical viability and practical considerations
}

\author{
Arulmozhi D Kandasamy, PhD*
}

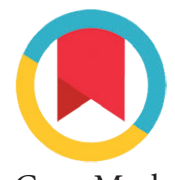

CrossMark

\section{ABSTRACT}

The U.S. Foodand Drug Administration and other regulatory bodies recently approved Praluent (alirocumab) and Repatha (evolocumab) for patients with hereditary forms of high cholesterol and those with cardiovascular disease. These drugs belong to a potent new class of injectable LDLlowering drugs known as PCSK9 inhibitors. Repatha was approved to treat patients with heterozygous familial hypercholesterolemia (HeFH) and patients with the rarer homozygous ( $\mathrm{HoFH}$ ) form of the disease. It was also approved for patients with cardiovascular disease including heart attack or stroke, who require additional cholesterol lowering. The scope of the approval was similar to the approval given to the Regeneron drug, Praluent (alirocumab), which was approved for patients with cardiovascular disease and those with $\mathrm{HeFH}$.

Keywords: PCSK9 inhibitors, Praluent ${ }^{\oplus}$, alirocumab, Repatha ${ }^{\circledR}$, evolocumab, LDL, HDL, cholesterol

*Correspondence to: Arulmozhi D Kandasamy, PhD, DiscoverSys Inc., Edmonton, Canada arul@discoversys.ca

Cite This Article: Kandasamy, A.D. 2017. PCSK9 inhibitors - Clinical viability and practical considerations. Diabesity 3(2): 5-8. D0I: 10.15562/ diabesity. 2017.39

\section{INTRODUCTION}

Statins have been the first-line drugs for lowering cholesterol since the late 1980s. They've been shown to prevent repeat heart attacks in people who have already had one and first heart attacks in a wide range of at-risk individuals. In about one in five people, though, a statin doesn't lower cholesterol enough. Adding a second drug that lowers cholesterol by a different mechanism doesn't always help. And some people can't take a statin because of side effects like muscle pain, liver damage, or the development of diabetes.

The U.S. Food and Drug Administration and other regulatory bodies recently approved Praluent (alirocumab) and Repatha (evolocumab) for patients with hereditary forms of high cholesterol and those with cardiovascular disease. These drugs belong to a potent new class of injectable LDL-lowering drugs known as PCSK9 inhibitors.

Repatha was approved to treat patients with heterozygous familial hypercholesterolemia $(\mathrm{HeFH})$ and patients with the rarer homozygous (HoFH) form of the disease. It was also approved for patients with cardiovascular disease including heart attack or stroke, who require additional cholesterol lowering. The scope of the approval was similar to the approval given to the Regeneron drug, Praluent (alirocumab), which was approved for patients with cardiovascular disease and those with $\mathrm{HeFH}$. The drugs are expected to generate more than $\$ 2$ billion a year each in sales by 2020 , according to Thomson Reuters data.

Praluent is given every other week by injection in doses of $75 \mathrm{mg}$ or $150 \mathrm{mg}$. Both doses of the drug will be priced wholesale at $\$ 1,120$ for a 28 -day supply, or roughly $\$ 14,560$ a year. After rebates, analysts expect the drug to cost about $\$ 12,400$ a year. Repatha is given as a $140 \mathrm{mg}$ injection every other week or as a monthly injection of $420 \mathrm{mg}$. Amgen said the wholesale cost of the drug will be $\$ 14,100$ a year for the every other week injection. The product will be available next week.

\section{PCSK9 INHIBITORS}

Monoclonal antibodies, target and inactivate a specific protein in the liver. Knocking out this protein, called proprotein convertase subtilisin kexin 9, dramatically reduces the amount of harmful LDL cholesterol circulating in the bloodstream. Lower LDL translates into healthier arteries and fewer heart attacks, strokes, and other problems related to cholesterol-clogged arteries. Proprotein convertase subtilisin/kexin type 9 (PCSK9) is an enzyme that plays an important role in lipid metabolism by modulating the density of LDL cholesterol receptors in multiple organs. The enzyme is synthesized in the nucleus, and after intramolecular autocatalytic cleavage of its $\mathrm{N}$-terminal prosegment in the endoplasmic reticulum, it is secreted from hepatocytes, where it binds to the surrounding LDL cholesterol receptors. The complex is then subject to endocytosis and degradation of its entire structure in lysosomes. ${ }^{7}$ This physiologic function leads to an inverse relationship between the level of PCSK9 in the blood and the number of LDL receptors; inhibition of PCSK9 prevents LDL receptor degradation within lysosomes and preserves receptor recycling 
to the hepatocyte surface. Each receptor normally recycles approximately 150 times. Thus, monoclonal antibody binding and inhibition of PCSK9 prevents PCSK9 binding to the LDL cholesterolLDL receptor complex and subsequent lysosomal degradation of the LDL receptor. The LDL-receptor recycling is preserved, with a consequent increase in receptor density on the hepatocyte surface and LDL cholesterol clearance.

\section{How do they work}

PCSK9 is predominantly produced in the liver. ${ }^{1-3}$ PCSK9 binds to the low density lipoprotein receptor (LDL-R) on the surface of hepatocytes, leading to the degradation of the LDL-R and higher plasma LDL-cholesterol (LDL-C) levels. ${ }^{4,5}$ Antibodies to PCSK9 interfere with its binding of the LDL-R leading to higher hepatic LDL-R expression and lower plasma LDL-C levels (figure 1). ${ }^{21}$

There are several strategies to lower free plasma PCSK9, including antisense, silencing ribonucleic acid (RNA), and monoclonal antibody strategies. PCSK9-antibodies are the first of these therapies approved for clinical use. These antibodies are specific for PCSK9 and do not bind to other members of the PCSK enzyme family.,

Alirocumab and evolocumab are fully humanized monoclonal antibodies that bind free plasma PCSK9, promoting degradation of this enzyme. ${ }^{9-12}$ As a result, less free PCSK9 is available in plasma to bind to LDL-R. This results in a higher fraction of LDL-R recycling towards the hepatocyte surface. As a direct consequence, the liver has the capacity to remove more LDL-C from the circulation, resulting in lower LDL-C plasma levels.

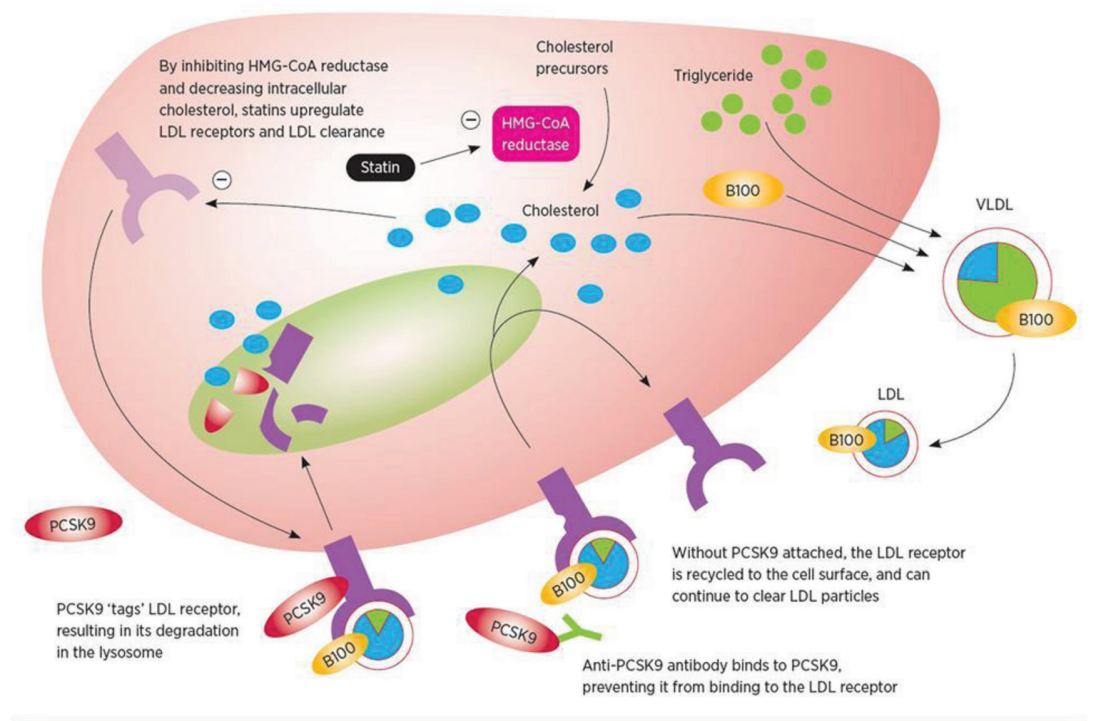

B100 apolipoprotein-B100 PCSK9 proprotein convertase subtilisin/kexin type 9 LDL low-density lipoprotein VLDL very low-density lipoprotein

Figure 1 Mechanism of PCSK9 inhibition (Image reproduced from Ref. 21)

\section{Evidence}

Results from seven multi-centre, double-blind, placebo- and/or active (ezetimibe)-controlled, randomized phase 3 trials evaluating evolocumab in more than 4,500 patients have been reported. ${ }^{13-19}$ The co-primary outcomes of five trials were the per cent change from baseline in LDL-C at 12 weeks and averaged between weeks 10 and 12 (to more accurately reflect average LDL-C reduction over the entire dosing interval). ${ }^{13-15,19}$ This bulletin reports data for the treatment difference at 12 weeks. The primary outcomes for the other two trials were per cent change from baseline in LDL-C at 12 weeks 41 or at 52 weeks. ${ }^{20}$

Five of the trials evaluated evolocumab administered at a dose of $140 \mathrm{mg}$ every two weeks or $420 \mathrm{mg}$ every month in patients with primary hypercholesterolemia (non-familial and heterozygous familial hypercholesterolemia) and mixed dyslipidemia. ${ }^{13-15,17,18} \mathrm{~A}$ heterogeneous population was assessed in these trials, in which approximately $20 \%$ had a history of coronary heart disease and fewer than $50 \%$ of participants were considered to be at moderate to high cardiovascular risk at baseline. One trial evaluated evolocumab administered at a dose of $420 \mathrm{mg}$ every month in patients with homozygous familial hypercholesterolemia. ${ }^{19}$ Approximately $43 \%$ of these patients had established coronary artery disease. One trial evaluated evolocumab at a dose of $420 \mathrm{mg}$ every month in patients with a range of cardiovascular risks. ${ }^{15}$ The majority (64\%) of participants in this trial were at low or moderate cardiovascular risk. Patients enrolled in the evolocumab phase 3 clinical program (who were not considered statin intolerant) were not all required to be taking maximally tolerated statin background therapy prior to the addition of evolocumab, which may not be well aligned with the proposed future use of evolocumab in clinical practice.

\section{Adverse Effects}

Overall, patients treated with PCSK9 inhibitors had higher rates of nonspecific side effects, such as arthralgia, headache, limb pain and fatigue, compared with placebo-treated patients. Adverse events were reported in 69 percent of people taking evolocumab in the clinical trials. Injection-site swelling or rash, limb pain, and fatigue were some of the reported side effects. Less than one percent reported mental confusion, difficulty focusing, or other neurocognitive issues. ${ }^{8}$

In the alirocumab trials, adverse events were reported in 81 percent of participants taking the drug. These included injection-site reactions, muscle pain, and eye-related events. Slightly more than 1 percent of participants reported 
neurocognitive adverse events. These included memory impairment and confusion. ${ }^{20}$

However, clinicians were most concerned about neurocognitive events. Overall, $1 \%$ to $1 \frac{1}{2} \%$ of patients experienced treatment-related neurocognitive effects, mainly confusion and some memory loss. However, it's important to note that the neurocognitive issues were not related to the amount of LDL-C decrease.

\section{CONCLUDING REMARKS}

PCSK9 inhibitor monoclonal antibodies have the potential to fill an important treatment gap in patients with harder-to-treat hypercholesterolemia. Results from trials, which mainly focus on surrogate markers of cardiovascular outcomes, showed that PCSK9 inhibitor monoclonal antibodies produce statistically significant reductions in LDL-C regardless of background lipid-lowering therapy. Based on the relatively short-term evidence that is currently available, these drugs appear to be safe, although the FDA has identified a potential signal for neurocognitive adverse effects. Given that cardiovascular disease is still one of the most important causes of death in Canada, despite the availability of statins for 25 years, PCSK9 inhibitor monoclonal antibodies are expected to be rapidly adopted if approved, particularly for patients with harder-to-treat hypercholesterolemia. However, long-term safety and the effect on cardiovascular outcomes remain to be established. Findings from long-term outcomes trials will not be available until 2017 or 2018 .

Given the potential broad clinical use of this new drug class, the high price, and the long time frame required for the introduction of less expensive subsequent entry biologics, the introduction of PCSK9 inhibitor monoclonal antibodies may potentially have a substantial budgetary impact on the Canadian health care system. Careful patient selection will be necessary. The requirement to self-administer the drug subcutaneously may also impact longterm patient acceptability and adherence. In order to determine the clinical impact and value of this new drug class on the potentially broad and diverse population of patients with difficult-to-treat hypercholesterolemia, the long-term safety, efficacy, and cost-effectiveness of PCSK9 inhibitor monoclonal antibodies need to be clarified. ${ }^{22}$

\section{DISCLAIMER}

This article is based on a report by Canadian Agency for Drugs and Technologies in Health. ${ }^{22}$

\section{REFERENCES}

1. Maxwell KN, Soccio RE, Duncan EM, et al. Novel putative SREBP and LXR target genes identified by microarray analysis in liver of cholesterol-fed mice. J Lipid Res 2003; 44:2109.

2. Seidah NG, Benjannet S, Wickham L, et al. The secretory proprotein convertase neural apoptosis-regulated convertase 1 (NARC-1): liver regeneration and neuronal differentiation. Proc Natl Acad Sci U S A 2003; 100:928.

3. Ferri N, Tibolla G, Pirillo A, et al. Proprotein convertase subtilisin kexin type 9 (PCSK9) secreted by cultured smooth muscle cells reduces macrophages LDLR levels. Atherosclerosis 2012; 220:381.

4. Zhang DW, Lagace TA, Garuti R, et al. Binding of proprotein convertase subtilisin/kexin type 9 to epidermal growth factor-like repeat A of low density lipoprotein receptor decreases receptor recycling and increases degradation. J Biol Chem 2007; 282:18602.

5. Lo Surdo P, Bottomley MJ, Calzetta A, et al. Mechanistic implications for LDL receptor degradation from the PCSK9/LDLR structure at neutral pH. EMBO Rep 2011; 12:1300.

6. Mullard A. Cholesterol-lowering blockbuster candidates speed into Phase III trials. Nat Rev Drug Discov 2012; 11:817.

7. McGovern TJ. Tertiary Pharmacology/Toxicology Review REPATHA (evolocumab) U.S. Food and Drug Administration 2015.

8. Chung JE. Clinical pharmacology review PRALUENT (Alirocumab). U.S. Food and Drug Administration 2014.

9. Stein EA, Swergold GD. Potential of proprotein convertase subtilisin/kexin type 9 based therapeutics. Curr Atheroscler Rep 2013; 15:310.

10. Manniello M, Pisano M. Alirocumab (Praluent): First in the New Class of PCSK9 Inhibitors. P T 2016; 41:28.

11. Chan JC, Piper DE, Cao Q, et al. A proprotein convertase subtilisin/kexin type 9 neutralizing antibody reduces serum cholesterol in mice and nonhuman primates. Proc Natl Acad Sci U S A 2009; 106:9820.

12. Stein EA, Mellis S, Yancopoulos GD, et al. Effect of a monoclonal antibody to PCSK9 on LDL cholesterol. N Engl J Med 2012; 366:1108.

13. Koren MJ, Lundqvist P, Bolognese M, Neutel JM, Monsalvo ML, Yang J, et al. Anti-PCSK9 monotherapy for hypercholesterolemia: the MENDEL-2 randomized, controlled phase III clinical trial of evolocumab. J Am Coll Cardiol. 2014 Jun 17;63(23):2531-40.

14. Robinson JG, Nedergaard BS, Rogers WJ, Fialkow J, Neutel JM, Ramstad D, et al. Effect of evolocumab or ezetimibe added to moderate- or high-intensity statin therapy on LDL-C lowering in patients with hypercholesterolemia: the LAPLACE-2 randomized clinical trial. JAMA. 2014 May 14;311(18):1870-82.

15. Kiyosue A, Honarpour N, Kurtz C, Xue A, Wasserman SM, Hirayama A. A Phase 3 Study of Evolocumab (AMG 145) in Statin-Treated Japanese Patients at High Cardiovascular Risk. Am J Cardiol. 2016 Jan 1;117(1):40-7. doi: 10.1016/j. amjcard.2015.10.021.

16. Blom DJ, Hala T, Bolognese M, Lillestol MJ, Toth PD, Burgess L, et al. A 52-week placebo-controlled trial of evolocumab in hyperlipidemia. N Engl J Med. 2014 May 8;370(19):1809-19.

17. Stroes E, Colquhoun D, Sullivan D, Civeira F, Rosenson RS, Watts GF, et al. Anti-PCSK9 antibody effectively lowers cholesterol in patients with statin intolerance: the GAUSS-2 randomized, placebo-controlled phase 3 clinical trial of evolocumab. J Am Coll Cardiol. 2014 Jun $17 ; 63(23): 2541-8$.

18. Raal FJ, Stein EA, Dufour R, Turner T, Civeira F, Burgess L, et al. PCSK9 inhibition with evolocumab (AMG 145) in heterozygous familial hypercholesterolaemia 
(RUTHERFORD-2): a randomised, double-blind, placebo-controlled trial. Lancet. 2015 Jan 24;385(9965):331-40.

19. Raal FJ, Honarpour N, Blom DJ, Hovingh GK, Xu F, Scott R, et al. Inhibition of PCSK9 with evolocumab in homozygous familial hypercholesterolaemia (TESLA Part B): a randomised, double-blind, placebo-controlled trial. Lancet. 2015 Jan 24;385(9965):341-50.

20. Robinson JG, Farnier M, Krempf M, Bergeron J, Luc G, Averna M, et al. for the ODYSSEY LONG TERM Investigators . Efficacy and Safety of Alirocumab in Reducing Lipids and Cardiovascular Events. N Engl J Med 2015; 372:1489-1499.

21. https://www.nps.org.au/australian-prescriber/articles/ pcsk9-inhibitors-mechanisms-of-action
22. https://www.cadth.ca/dv/pcsk9-inhibitor-monoclonalantibodies-treatment-hypercholesterolemia

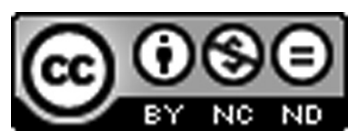

This work is licensed under a Creative Commons Attribution-Non Commercial-No Derivatives 4.0 International License. To view a copy of this license, visit http://creativecommons.org/licenses/by-nc-nd/4.0/ 